\title{
Decisions and Infrastructure (In)visibility: A Case Study
}

\author{
Roberta Cuel and Diego Ponte
}

\begin{abstract}
This work focuses on how digital infrastructures of a complex interorganizational system becomes visible and changes. While scientific research on infrastructures have addressed both theoretical and methodological issues, the way in which an inter-organizational and complex infrastructure is shaped and "cultivated" remains unexplored. The aim of this paper is to describe the most significant elements that characterize the interplay between human decisions and behaviors, infrastructure innovation and its visibility. These have been used as requirements to create a decision support system that could help experts to take decisions on an infrastructure for a planned change. In the paper, a longitudinal analysis is proposed with a focus on changes planned and implemented in the Air Traffic Management (a complex inter-organizational system adopted in all the European countries).
\end{abstract}

Keywords Digital infrastructure - Decisions - Socio-technical system - Air traffic management $(\mathrm{ATM}) \cdot$ Longitudinal analysis

\section{Introduction}

A large body of literature on infrastructures has studied both theoretical and methodological issues of their visibility or invisibility and the role played by work practices, individual habits, and organizational cultures [20, 23]. The way in which an inter-organizational and complex infrastructure is shaped and "cultivated", however, remains unexplored. In this paper, authors analyze the changes implemented in a complex inter-organizational system and its (in)visible infrastructure unveiling different elements that may shape and modify the infrastructure itself. These

\footnotetext{
R. Cuel $(\varangle) \cdot$ D. Ponte

Department of Economics and Management, University of Trento, Via Inama, 5,

38122 Trento, Italy

e-mail: roberta.cuel@unitn.it

D. Ponte

e-mail: diego.ponte@unitn.it 
are actors, organizational culture, processes, technology, and artefacts. In the case planned changes occur, a multitude of decisions and negotiations actions are taken on the interconnections and interdependencies of people, activities, structures and cognitive elements.

These changes are investigated in the case of European Air Traffic Management (thereafter ATM), the complex inter-organizational infrastructure, which assists the flight of an aircraft when departing, cruising, and landing at an airport. This is accomplished through distinct activities such as air traffic control and air traffic flow management. ATM services are complex systems because they exploit advanced technologies and require highly skilled human resources entailing significant investment in personnel, assets and training. Moreover, they are endow by different national and international organizations all over the Europe.

The analysis is carried out using qualitative research made up of semi-structured interviews, focus groups with experts from the sector and review of documents and reports [40]. The conclusion outlines the elements and categories of the decision processes that come into play when creating, maintaining or changing an infrastructure. These were used to create a decision support system that could help ATM experts to represent a domain and its underlying infrastructure (from the perspective of an organizational unit/function), simulate a change, reason on its consequences on other functional areas (other unit infrastructures), and finally take a decisions, namely plan a change of a global or inter-organizational infrastructure.

The following section introduces the literature review on infrastructure (in)visibility and its dynamics. The third and fourth sections present the case study and the research method. Thereafter the results are illustrated. The last section sketches out the theoretical implications of this research on software requirements for the development of a decision support system.

\section{Infrastructures, (In)visibility and Decision Making}

Among others, one common definition of sociotechnical infrastructure is that it is a robust network of people, artefacts, and institutions that generate, share and maintain specific knowledge about the human and natural worlds [14]. A large body of literature-from interactionism to the workplace studies about infrastructures, has stressed the important role played by the human elements of infrastructures such as work practices, individual habits, and the organizational culture $[2,7,15,18,28,35$, 36].

Infrastructures shape what and how actors understand and interpret their world through practices, routines and organizational cultures, informational and knowledge infrastructures [9]. Infrastructures exist in the background, are invisible and are takenfor-granted by actors who perform routines and practices [7].

An infrastructure is generally invisible in daily life and operates below the surface but becomes visible in two main cases [23]. 
1. When it breaks down [7]. When a server goes down, a bridge is washed out, or when a power blackout occurs, the infrastructure becomes evident to the actors that use it. The safe management of such situations implies the creation and implementation of ex-ante and ex-post procedures such as back-up mechanisms or other emergency procedures, which should fix breakdowns and bugs.

2. When it is analyzed during meetings (as in a "sensemaking" process) that aim to create, maintain or change an infrastructure [7, 21, 24]. Visibility of an infrastructure is very much intertwined with the change of an infrastructure. When the infrastructure is complex, the changing process become an extremely complex venture. It is not an instantaneous process; it requires time and iterative development, involves multiple actors and implies various non-deterministic phases $[1,16,19]$.

Since the infrastructure supports and is, in turn, inhabited by social, political and technical rudiments, its creation or change cannot be analyzed only from a technological point of view but rather from the result of the actors' decisions, negotiations on practices, routines, assets and the sociotechnical elements that make up the infrastructure itself. Previous research [7, 29] has shown that two important characteristics are linked to the (in)visibility of infrastructures:

1. The infrastructure is the result of negotiation among heterogeneous actors.

2. People is connected to activities, structures and cognitive elements embedded in an infrastructure.

As such, decision processes in complex organizations represent one of the most important activities [39] for changing, cultivating, and making visible infrastructures.

According to Beersma and De Dreu [4, 11], group work involves negotiations, then negotiation dynamics have a prominent role in decision making, and finally decisions are closely linked to the knowledge of individuals, their ability to share and the common knowledge infrastructure they rely on.

Various elements are considered antecedents in negotiation and decision processes that shape infrastructures and may be used to make "visible" the infrastructures themselves. Among others: skills, knowledge and competencies [27]; procedures, routines and rules [3]; roles, power and social motives [17, 26]. The importance of these elements emerges even more forcefully when the organization is large and complex.

The goal of our work is to investigate how an inter-organizational and complex infrastructure is shaped and "cultivated" once a change is planned and implemented in a complex inter-organizational system. In the interplay between infrastructure and its (in)visibility decision an negotiation have an important role and an impact on actors perception, organizational and inter-organizational culture, processes, technology, artefacts, etc. (Fig. 1). These elements will be investigated in the following case study. 
Fig. 1 The goal of the research

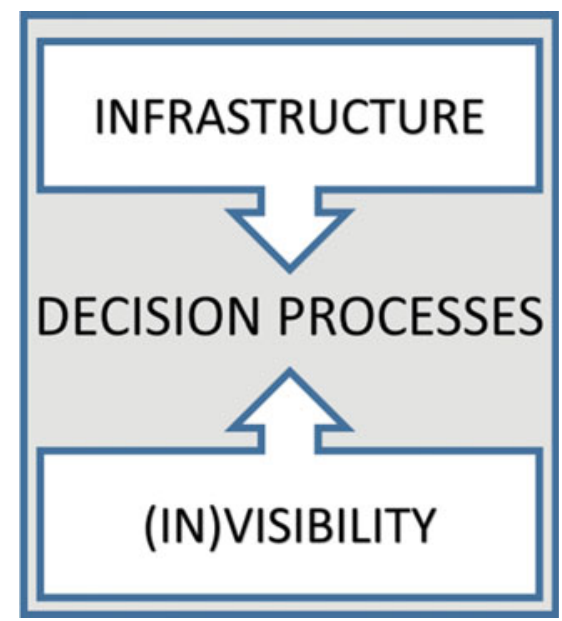

\section{The Case Study}

Air Traffic Management (thereafter ATM) is the entire ecology of systems that assist the flight of an aircraft - departing, cruising, and landing at an airport [12]. The European Organisation for the Safety of Air Navigation (EUROCONTROL) manages and controls - in cooperation with national bodies of EU Nations - the air traffic across Europe. Two main elements make ATM a very complex infrastructure. First, ATM is an inter-organizational system currently populated by a set of heterogeneous actors:

- air navigation service providers (e.g. DFS in Germany and ENAV in Italy)

- European Civil Aviation Conference member states

- civil and military experts in airspace design

- passengers and airspace users

- flight planner organisations

- relevant international bodies.

Second, since one of the main goals is the safety of flights, ATM and the interaction among actors is driven by strict national and international regulation that formalise the working procedures. Therefore, the ATM infrastructure is quite rigid and any change is a complex endeavour that affects hundreds of national and international organizations, actors, procedures, assets and is subject to many regulations aimed at assuring flight safety and security.

Such complexity is particularly evident in the Single European Sky initiative, an EU initiative that has the goal to design and manage the evolution of the airspace in EU toward the creation of a unique regulator for ATM within EU. Such project, started in 2001, is still running and its efforts to change the infrastructure are described in the following section. 


\subsection{Background: Willingness to Make ATM More Efficient in Europe}

The current configuration of European ATM is the result of the harmonization process in European countries implemented by the EU in the 1960s. The foundation of EUROCONTROL is the visible element of this effort.

Twenty years ago, the EU introduced the Single European Sky (SES) initiative with the goal of improving operational efficiency of ATM designing, managing and regulating a single coordinated airspace throughout the European Union. European airspace is one of the busiest in the world but the current system of ATM suffers from inefficiencies, such as the boundaries of air traffic control that follow national borders, and having large areas of European airspace reserved for military use. ATM relies on a number of new key features including better trajectory management, new aircraft separation modes and full integration of airport operations. The full initiative is an EU collaborative research programme called Single European Sky ATM Research (SESAR) and it is intended to last several decades through three phases [38]. Considering the complexity of the project and the numerous initiatives underway, this paper focuses on only one of these issues, namely Air Traffic Control (ATC) activity aimed at assisting aircraft in the upper airspace; one of the most critical activities, it is further described below.

\subsection{The Starting Point: Sectored Air Traffic Control}

The duty of ATC is to organize air traffic flow, to prevent collisions between aircraft and to provide pilots with information. Controllers apply separation rules to keep aircraft at a safe distance from each other to reduce the risk of collisions or other types of accidents (e.g. wake turbulence) and move all aircraft safely and efficiently through their assigned sector of airspace as well as on the ground. Managing the traffic flow, balancing the demand and capacity of the airspace, and preventing collisions is a complex service involving organizational, cognitive, structural and technological issues. One of the most important is the management of complexity. Diverse organizational, technological and structural solutions have been adopted to manage complexity when controlling aircraft. One solution adopted for ATC in Europe is the partitioning of the airspace into geographical sectors. Each airspace passing through a sector is controlled by a specific organization or Air Control Centre (ACC). In each sector, a pool of controllers perform different activities:

- Take care of and interact with pilots of the aircraft flying within the sector.

- Coordinate with controllers of other sectors to define the specific paths to bridge sectors (Fig. 2, left).

One of the main limitations of this type of work setting is that an increase in the air traffic flow means an increase on the workload for the air traffic controllers. 
In particular, the coordination efforts between sectors increase significantly [5]. A common practice used to reduce this workload excess has been to decrease the size of the sectors thus creating more sectors. Unfortunately, such practice displays limitations:

1. A smaller sector means that controllers may exert less tactical and strategic control on aircraft.

2. Partitioning the airspace cannot be done indefinitely. Physical limitations do not allow partitioning the airspace indefinitely. This problem is already present in some European countries [6, 37].

Over the last two decades, different solutions have been proposed and scrutinized to overcome the limitations of the traditional sector-based control system; one of the most explored is the Sectorless scenario.

\subsection{The Proposed Change: The Sectorless Scenario}

For the last two decades, international bodies, practitioners and scholars in the sector have discussed an innovative approach to controlling airspace: the Sectorless scenario $[5,12,13,34]$. The Sectorless scenario envisages air traffic control without the conventional geography-based sectors. This new approach means that several aircraft are assigned to a single controller regardless of their location. Each single controller guides the aircraft during its entire flight in upper airspace (Fig. 2, right).

The Sectorless scenario is said to offer significant improvements while addressing the main bottlenecks of the traditional sectored approach. The main foreseen improvements can be summarised as [6, 22, 37]:
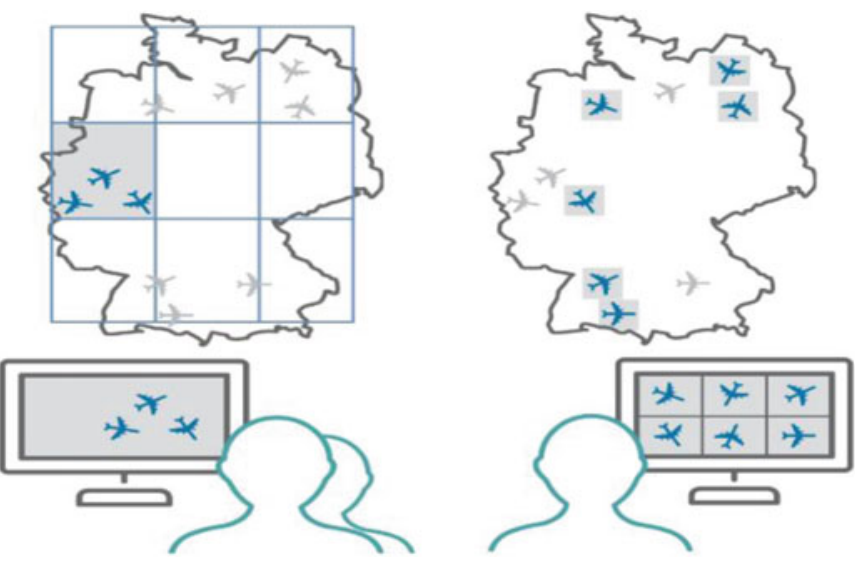

Fig. 2 Sectored versus Sectorless control scenario Source [13] 
- A higher number of air traffic flights: the system is able to control a greater number of flights.

- Less workload: controllers face less workload and also less handovers.

- Efficiency in terms of costs and time: Sectorless allows for more linear trajectories meaning less fuel and less travel time for each flight.

- A single point of contact for pilots: when entering a Sectorless area pilots have a sole controller to talk to.

In order to assess the feasibility of this concept, over the last decade scholars have focused on several operative aspects of the Sectorless scenario including the change in controllers' tasks, the assignment procedures of aircraft, the priority rules and the safety assessment routines $[6,25]$. Since the Sectorless scenario is a complex innovation, its implementation will last for several years to become gradually operational over the next ten years, more than a decade since the initial exploration of the concept. The technical, organizational, economic and procedural innovations of the scenario imply numerous changes within the sector as a result of decisions to plan and implement changes to the infrastructure and its interconnected practices.

\subsection{Research Method}

The work was organised in the following phases. First, the review of documents of official ATM reports and scientific papers describing innovation and changes in ATM and, more specifically, in air traffic control systems. Second phase, semi-structured interviews: 4 ATM experts were interviewed to identify the most important decision process categories that affect changes in ATM. Third phase, one-day focus groups took place in June 2016 and March 2017. Table 1 briefly describes the experts' roles and competences.

All the interviews and the focus groups were recorded and transcribed. The narrative data was organized into elements and analytical categories. In particular, the interviews were aimed at identifying the most significant antecedent elements and emerging categories in the decision processes on change and innovation of the ATM infrastructure and of flight control systems. The categories identified during the preliminary interviews, were verified with the discussion in the focus groups [40].

\section{Data Analysis}

In the analysis of all documents, interviews, and focus groups five of the most significant key elements that characterize decision processes within ATM systems and which may influence the infrastructure (in)visibility are uncovered. Each element (Table 2) was analysed in depth and various analytical categories emerged [33]. The 
Table 1 Expertise of the experts participating in the focus groups

\begin{tabular}{l|l}
\hline Role & Competences \\
\hline ATM Security Expert & $\begin{array}{l}\text { Supports national service providers, state authorities and } \\
\text { the industry with respect to ATM security; works for an } \\
\text { international organization providing ATM services }\end{array}$ \\
\hline Senior Enterprise Architect & $\begin{array}{l}\text { Supports the strategic development of Air Traffic } \\
\text { Management; works for a European National Service } \\
\text { Provider }\end{array}$ \\
\hline ATM Safety Expert & $\begin{array}{l}\text { Expert on human resource within ATM; works for a } \\
\text { European National Service Provider }\end{array}$ \\
\hline Manager of an ATM R\&D team & $\begin{array}{l}\text { Expert in security and safety; works for a European } \\
\text { National Service Provider }\end{array}$ \\
\hline Head of the research unit & $\begin{array}{l}\text { Expert in process reorganization and innovation in the ATM } \\
\text { system; works for a European National Service Provider }\end{array}$ \\
\hline Senior researcher & $\begin{array}{l}\text { National Service Provider } \\
\text { Expert in communication, navigation and surveillance; } \\
\text { works for a research unit of a European National Service } \\
\text { Provider }\end{array}$ \\
\hline
\end{tabular}

Table 2 Expertise of the experts participating in the focus groups

\begin{tabular}{l|l}
\hline Element & Categories \\
\hline The actor involvement & Play a role; Actor engaging; Doing cultures \\
\hline Dealing with the problem/issue & Objectifying the problem \\
\hline Solving the conflicts & Acting on procedures and artefacts; Mastering in command \\
\hline Driving the decision process & Motivating socially \\
\hline The levels of decision process & $\begin{array}{l}\text { Handling events; Changing procedures; Crossing the } \\
\text { boundaries }\end{array}$ \\
\hline
\end{tabular}

following describes only the elements and then outlines whether and to what extent these elements are embedded in a decision making tool.

\subsection{The Actors Involvement}

As explicated during the interviews, actors play different roles while dealing with decision-making processes. Therefore, the "play a role" category has been unveiled. It identifies the position actors have within ATM and the situation they encounter while participating. The role played by each actor is inevitably influenced by individual motivation and level of engagement so "actor engaging" is another key category. The role played and the type of engagement are, however, closely related to the existing organizational culture within the ATM system. Thus, "doing culture" category 
explain how decisions affect other actors. This is evident when reading one of the interviewees' words: "There must be a proactive debate among the various actors around the table and there must be no hypersensitivity. This is part of a culture, which means creating a solid organizational culture [...]".

\subsection{Dealing with the Problem/Issue}

The problem/issue or subject of the decision process often appears to be a set of unresolved secondary and often subjective issues that contaminate the real problem to be decided. For this reason, a decision process may be carried out over a very long term, and should involve various actors with different views and approaches.

The category "objectifying the problem" is represented by these words from an interviewee: "[...] first of all the presentation of the problem. It must be presented in as objective a way as possible, because usually the problem comes contaminated. [...]".

Knowledge has to be cleaned to clearly represent a problem or an issue at stake. In other words, the problem is usually described from the expert's point of view, but in order to make a more objective decision involving various actors, the problem should be clearly described using common language and common values.

\subsection{Solving the Conflicts}

Conflicts may occur during decision processes for different reasons such as conflicting interests and motivations or gaps in the process. A common reason for conflicts is having "contaminated information" which may make actors bias in favour of a specific interest. In case of conflict, the decision makers must consider various elements in an attempt to reach a common decision: the actors themselves, the procedures and the artefacts involved. The category "mastering in command" can be represented by the following sentence provided by an interviewee: "[...] There must be the master in command when an unforeseen problem occurs that has an effect on a decision [...]". In other words, $w$ hen a dialectic process arises and the conflict cannot be solved, the presence of a master in command actor drives the whole decision process is required.

\subsection{Driving the Decision Process}

A decision may affect the balance within the system and favour the interests of one side or another. In this complex system, the above-mentioned elements are interwoven with power, interests and social motives, and drive decision processes. Another category identified is "motivating socially". Social motives seem to play a prominent 
role, especially if related to reputation, confidence and trust within any hierarchical structure.

\subsection{The Levels of Decision Process}

The analysis of the collected data allowed highlighting three levels at which decisions are made, namely operational, managerial and strategic.

The operational level deals with the daily management of any air traffic action, and decisions are made in real-time. The category identified is defined as "handling events". Event management should be proactive but in most cases, the management places the guilt on the single individual leader (the master in command).

The managerial level deals with any technical change that may occur during a revision of ATM procedures, such as the introduction of new technologies, protocols etc. The changes are usually planned and are based on in depth technical and specialized knowledge shared in national and multinational projects. Thus, the authors identified a category called "changing procedures".

Changes in complex inter-organizational systems must necessarily take into account a variety of aspects; those linked to the actors involved (particularly stakeholders), those related to the economics and, no less important, those linked to the political elements. The strategic practices deal with the adoption of policies, norms and regulations at national and international levels. The category identified in this level is "crossing the boundaries" as decisions must necessarily take into account different contexts across national and international boundaries.

\section{Discussion on Infrastructure (In)visibility}

From the collected data, five elements and ten analytical categories were identified (Table 2). The relationships that forms the sociotechnical infrastructure emerges as the result of negotiations between actors and the role they play (even in terms of power) in the decision processes. Actors involved in the decision processes attempt to "clean" the information from contamination in order to share the most objective and comprehensive information, thus making visible the infrastructure and introducing new changes. Often the negotiation is not an easy process because actors belong to different organizations that operate in a complex inter-organizational system and decisions are often taken "acting on" human actors, procedures and/or artefacts. Experts can play the role of masters in command because of their skill sets, expertise and reputation in the entire organizational system. Decision processes on infrastructures go through three levels, namely operational, managerial and strategic which all have different effects on the infrastructure (in)visibility (Fig. 3).

These results are used to develop a decision support system called PACAS Platform (Fig. 4). It enables ATM experts to: (i) represent a domain and its underlying 


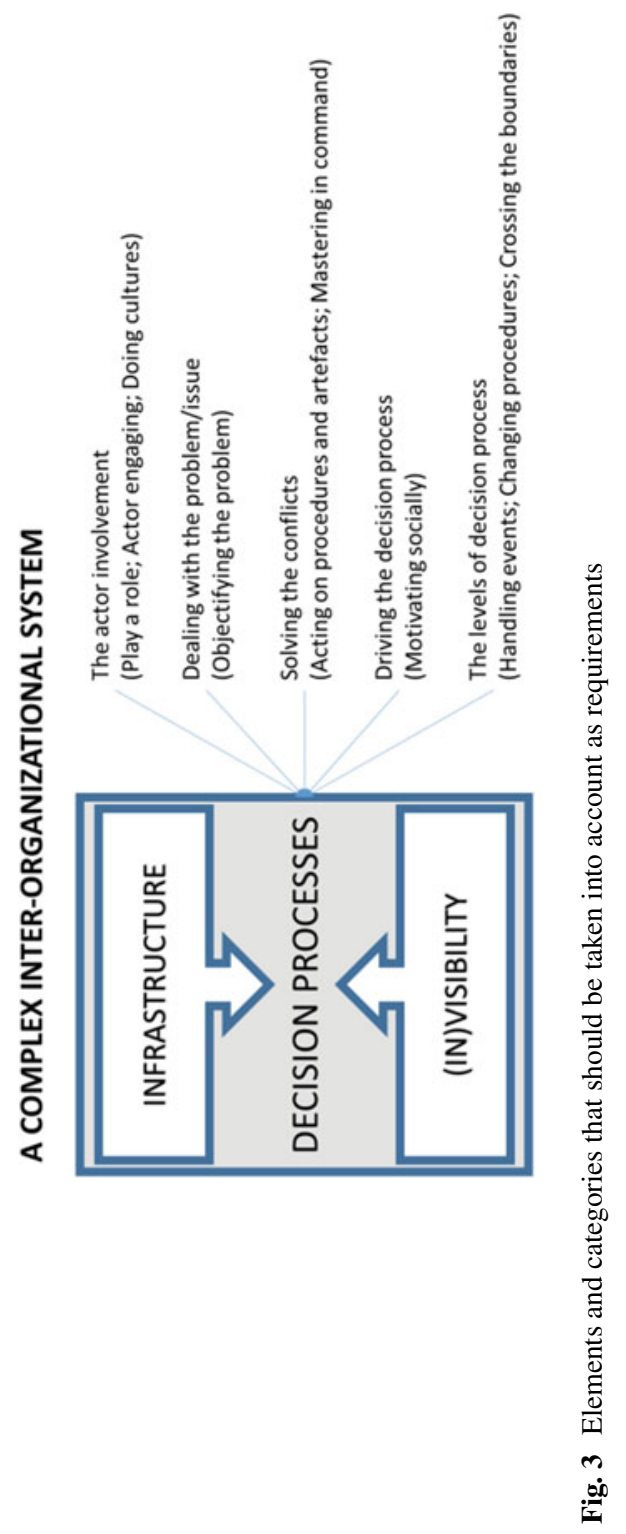




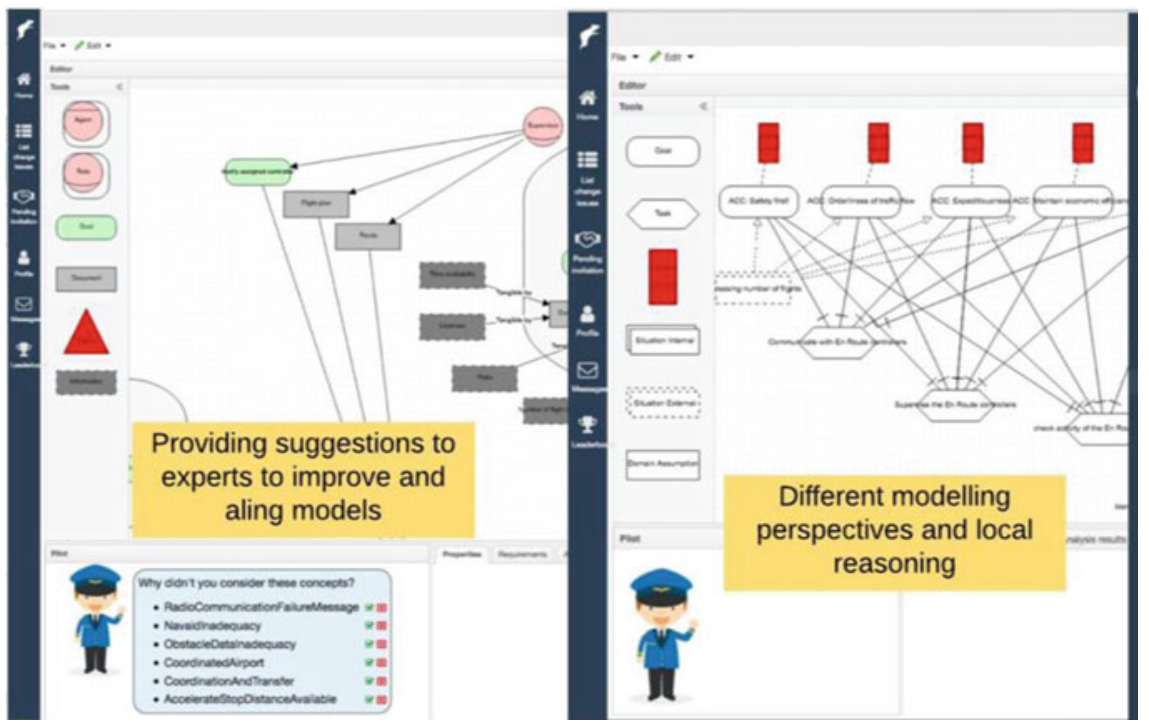

Fig. 4 1st release of the Pacas Platform

infrastructure (from the perspective of an organizational unit/function); (ii) simulate a change; (iii) reason on its consequences on the representation model of other functional areas (other unit infrastructures); (iv) take a decisions, namely plan a change of a global or inter-organizational infrastructure [30, 31].

In other words, the goal of the PACAS platform is to allow ATM domain stakeholders to take decisions for change management improving air transportation performance aspects such as safety, capacity, security, while ensuring cost efficiency and cutting down environmental impacts $[8,10]$.

The idea, is that actors, directly involved in the decision processes, play a specific role in both the real life and in the platform, and make the infrastructure visible because, through the negotiation of interests, power and strategies, they use and at the same time may influence the inter-organizational culture and reveal the infrastructure underlying the entire ATM system. The actor engagement is carried out through gamification processes and gamified roles (such as the game master) in which users are involved [32]. In order to allow users to deal with their issues in a proper way, a modelling language based tool and a multi-view approach have been developed allowing each user to focus on her own individual perspective, without the need of a holistic representation, and to negotiate with the others what really matters and what really should be objectified. Conflicts are solved acting on procedures, unveiling connection between different views (through automatic reasoning), or asking the master in command to take a decision. The control of the process and the action taken in the platform push actors to get socially motivated and to take a decision in a reasonable period. The different roles planned in the platform enable users to handle 
events, change procedure and cross boundaries acting at different levels of decision processes.

This paper has shown how the elements that characterize group decisions contribute to ATM infrastructures (in)visibility and how these can be embedded in a Decision Support System.

Acknowledgements This work has received funding from a grant agreement under European Union Horizon 2020 research and innovation programme.

The authors wish to thank Dr. Giusi Orabona for her contribution on a previous version of the paper.

\section{References}

1. Aanestad, M., et al. (2014). Infrastructuring work: Building a state-wide hospital information infrastructure in India. Information Systems Research, 25(4), 834-845.

2. Argyris, C. (1973). Personality and organization theory revisited. Administrative Science Quarterly, 18(2), 141.

3. Bazerman, M., et al. (1991). Negotiator rationality and negotiator cognition: The interactive roles of prescriptive and descriptive research. Negotiation Analysis, 109-130.

4. Beersma, B., \& De Dreu, C. K. (2002). Integrative and distributive negotiation in small groups: Effects of task structure, decision rule, and social motive. Organizational Behavior and Human Decision Processes, 87(2), 227-252.

5. Birkmeier, B., et al. (2016). Controller team possibilities for sectorless air traffic management. In Integrated Communications Navigation and Surveillance (ICNS), 6C3-1, IEEE.

6. Birkmeier, B., et al. (2014). Five transition strategies for sectorless ATM. In Digital Avionics Systems Conference (DASC), 2014 IEEE/AIAA 33rd. IEEE.

7. Bowker, G. C., Star, S. L. (1999). Sorting things out: Classification and its consequences. MIT Press.

8. Chopra, A. K., et al. (2011). Sociotechnical trust: An architectural approach. In International Conference on Conceptual Modeling. Heidelberg: Springer.

9. Constantinides, P., \& Barrett, M. (2014). Information infrastructure development and governance as collective action. Information Systems Research, 26(1), 40-56.

10. Dalpiaz, F., et al. (2016). Security requirements engineering: Designing secure socio-technical systems. MIT Press.

11. De Dreu, C. K., et al. (2000). Influence of social motives on integrative negotiation: A metaanalytic review and test of two theories. Journal of Personality and Social Psychology, 78(5), 889.

12. Duong, V., et al. (2002). Sector-less air traffic management: Initial investigations. Air Traffic Control Quarterly, 10(4), 379-393.

13. Duong, V., et al. (2001). Sectorless air traffic management. In 4th USA/Europe Air Traffic Management $R \& D$ Seminar.

14. Edwards, P. (2010). A vast machine: Computer models, climate data, and the politics of global warming. MIT Press.

15. Edwards, P. N. (2003). Infrastructure and modernity: Force, time, and social organization in the history of sociotechnical systems. Modernity and Technology, 1, 185-226.

16. Gasson, S. (2006). A genealogical study of boundary-spanning IS design. European Journal of Information Systems, 15(1), 26-41.

17. Giebels, E., et al. (2000). Interdependence in negotiation: Effects of exit options and social motive on distributive and integrative negotiation. European Journal of Social Psychology, $30(2), 255-272$.

18. Heath, C., \& Luff, P. (2000). Technology in action. Cambridge: Cambridge University Press. 
19. Horton, K. S., \& Wood-Harper, T. A. (2006). The shaping of I.T. trajectories: Evidence from the U.K. public sector. European Journal of Information Systems, 15(2), 214-224.

20. Iannacci, F. (2010). When is an information infrastructure? Investigating the emergence of public sector information infrastructures. European Journal of Information Systems, 19(1), $35-48$.

21. Jackson, S. J. (2014). 11 rethinking repair. Media technologies: Essays on communication, materiality, and society, pp. 221-239.

22. Kaltenhaeuser, S., et al. (2017). A concept for improved integration of Space Vehicle Operation into ATM. In 33rd Space Symposium.

23. Karasti, H., et al. (2010). Infrastructure time: Long-term matters in collaborative development. Computer Supported Cooperative Work, 19(3-4), 377-415.

24. Karasti, H., et al. (2016). Special issue on knowledge infrastructures. Science \& Technology Studies, 29, 1-4.

25. Korn, B., et al. (2009). Sectorless ATM-A concept to increase en-route efficiency. In DASC'09. IEEE/AIAA 28th. IEEE.

26. Mannix, E. A. (1993). Organizations as resource dilemmas: The effects of power balance on coalition formation in small groups. Organizational Behavior and Human Decision Process, $55(1), 1-22$.

27. Maznevski, M. L. (1994). Understanding our differences: Performance in decision-making groups with diverse members. Human Relations, 47(5), 531-552.

28. Mongili, A., \& Pellegrino, G. (2014). Information infrastructure(s): Boundaries, ecologies, multiplicity. Cambridge: Cambridge Scholars Publishing.

29. Neumann, L., et al. (1996). Making infrastructure: The dream of a common language. PDC.

30. PACAS Consortium: D4.2 First Release of the Reasoning Proof-of-Concept. (2017).

31. PACAS Consortium: D5.2 Validation Report. (2018).

32. Piras, L., et al. (2017). Gamification solutions for software acceptance: A comparative study of Requirements Engineering and Organizational Behavior techniques. In Proceedings - International Conference on Research Challenges in Information Science.

33. Ponte, D., et al. (2018). Decision making processes and visibility of a global infrastructure: The air traffic management. In Workshop di Organizzazione Aziendale. Rome.

34. Riviere, T. (2004). Redesign of the European route network for sector-less. In ICRAT 2004, 1st International Conference on Research in Air Transportation.

35. Schein, E. H. (1980). Organizational psychology (pp. 177-178). Englewood Cliffs, NJ Repr. by Permis. Prentice-Hall., Inc., Englewood Cliffs, NJ.

36. Schmidt, K., \& Bannon, L. (2013). Constructing CSCW: The first quarter century. Computer Supported Cooperative Work, 22(4-6), 345-372.

37. Schmitt, A. R., et al. (2011). Balancing controller workload within a sectorless ATM concept. CEAS Aeronautical Journal, 2(1-4), 35-41.

38. SESAR: SESAR Joint Undertaking. https://www.sesarju.eu/approach. Last accessed January 30, 2019.

39. Simon, H. (2000). Barriers and bounds to rationality. Structural Change and Economic Dynamics, 11(1-2), 243-253.

40. Yin, R. (2003). Case study research, design and methods. Thousand Oaks, California: Sage Publications Inc. 